\title{
The Importance of Clinical History Taking when Assessing Patient Suspected Positive COVID-19
}

\author{
Zainul Ikhwan Ahmad Khusairi, Kartik K, Ahmad Bilal AN, Chung WM \\ Emergency and Trauma Department, Taiping Hospital, Perak DarulRidzuan, Malaysia \\ DOI: http://dx.doi.org/10.31344/ijhhs.v5i0.304
}

Introduction: COVID-19 pandemic is an on-going devastating global event. It starts from December 2019 and is yet to resolve. To date, there are more than 25 million people diagnosed and 850 thousand deaths with COVID-19 (CDC, 2020). Healthcare services throughout the world are facing immense challenges. History taking has been inevitably the most emphasized tool in approaching all patients. However, the usefulness of medical history strongly depends on the patient's story offered. Incorrect or hidden history may not only bring wrong diagnosis but endanger health care personnel.

Objectives: This case series is aimed to describe the importance of clinical history-taking in assessing patients associated with COVID-19 symptoms or history.

Methods: Assessment through interview method in taking patient history was used. The focus of history taking was related to health and COVID-19 history. The data collected is a type of secondary data from medical reports of patients who come to receive medical services at the Emergency and Trauma Department, General Public Hospital Taiping, Perak. A total of three cases are taken using the purposive sampling technique. Data is analysed and presented in the form of qualitative data.

Results: This study found that the clinical history taking through interview method has successfully identified three patients with positive COVID-19 through assessment conducted.

Conclusion: Therefore, the study proved that effective history taking implemented by paramedics was able to identify patients with positive COVID-19 and to plan an appropriate management and help them get discharged without complications. All physicians and other health care practitioners in the Emergency Department or in the clinical ward are also advised to be more competent by increasing their level of knowledge and skills related to assessing patients through effective clinical history taking.

Keywords: clinical history taking, COVID-19, severe acute respiratory illness (SARI), emergency department 